\title{
ПАНГИПОПИТУИТАРИЗМ КАК ПЕРВОЕ ПРОЯВЛЕНИЕ ГЕНЕРАЛИЗОВАННОГО САРКОИДОЗА (КЛИНИЧЕСКИЙ СЛУЧАЙ)
}

\author{
Уханова Ю.А., Иловайская И.А.
}

ГБУЗ МО «Московский научно-исследовательский клинический институт им. М.Ф. Владимирского», Москва

Саркоидоз - это системное воспалительное заболевание неизвестной этиологии, характеризующееся образованием неказеифицирующихся гранулём, мультисистемным поражением различных органов. Наиболее часто отмечается поражение легких, поражение центральной нервной системы (нейросаркоидоз) встречается у 5-15\% пациентов, из них гранулематозная инфильтрация гипофиза и гипоталамуса наблюдается лишь у $2-8 \%$ пациентов.

ЦЕЛЬ: продемонстрировать случай саркоидоза, дебютировавшего симптомами гипопитуитаризма в результате образования неказеифицирующей гранулемы в гипоталамической области.

МАТЕРИАЛЫ И МЕТОДЫ:Пациент М., 40 лет, считал себя больным с 25 лет, когда появились и постепенно прогрессировали общая слабость, снижение либидо, эректильная дисфункция, отметил уменьшение оволосения на лице. Впервые обследован в возрасте 27 лет, выявлены гипогонадотропный гипогонадизм и гиперпролактинемия, на МСКТ головного мозга - очаг в гипоталамо-гипофизарной области размерами 2,5x4 мм, назначена терапия тестостероном и агонистами дофаминовых рецепторов. Состояние улучшилось, уровни тестостерона и пролактина нормализовались. В 32 г. стали беспокоить слабость, отсутствие аппетита, снижение АД, жажда и обильное мочеиспускание. При обследовании диагностированы вторичный гипотиреоз, вторичная гипокортицизм и несахарный диабет. По данным МРТ - увеличение размеров образования гипоталамуса до 12x11x9 мм. Была скорректирована заместительная гормональная терапия, пангипопитуитаризм и несахарный диабет были компенсированы. Состояние улучшилось, однако продолжали беспокоить жалобы на слабость, повышенную утомляемость.

РЕзУЛЬТАТЫ:в 36 лет по данным МРТ отмечено увеличение образования гипоталамуса до 15×12×11 мм. При флюорографии обнаружены очаги поражения легочной ткани, в связи с чем выполнено КТ органов грудной клетки: характерные для саркоидоза признаки поражения легких, внутригрудных лимфатических узлов, периферических лимфатических узлов. При осмотре на кожных покровах отмечены множественные бугорки фиолетового цвета до нескольких сантиметров в диаметре. При активном расспросе пациент отметил, что такие бугорки периодически появлялись на коже с 28 лет при длительном пребывании на солнце, но пациент не обращал внимания. После дообследования установлен диагноз: «Саркоидоз легких, внутригрудных лимфатических узлов, периферических лимфатических узлов, ЦНС, ЛОР-органов, печени, морфологически неверифицированный». Было назначено лечение метотрексатом, метилпреднизолоном по поводу саркоидоза и продолжена заместительная гормональная терапия по поводу гипопитуитаризма и несахарного диабета. На фоне лечения саркоидоза, состояние пациента улучшилось, через год (в 37 лет) по данным МРТ отмечен регресс образования гипоталамической области, что подтвердило диагноз нейросаркоидоза.

ВЫВОдЫ: в связи с формированием саркоидной гранулемы в гипоталамической области, в дебюте саркоидоза у пациента отмечались симптомы гипопитуитаризма, что является редким вариантом развития заболевания. На фоне адекватно подобранной заместительной гормональной терапии по поводу пангипопитуитаризма и несахарного диабета у пациента сохранялись жалобы на слабость и утомляемость, что в дальнейшем оказалось проявлениями саркоидоза.

КЛЮЧЕВЫЕ СЛОВА: пангИпопитуитаризм; саркоидоз; нейросаркоидоз; саркоидная гранулема. 